\title{
Quantitative modelling of the closure of meso-scale parallel currents in the nightside ionosphere
}

\author{
A. Marchaudon ${ }^{1}$, J.-C. Cerisier ${ }^{1}$, O. Amm ${ }^{2}$, M. Lester ${ }^{3}$, C. W. Carlson ${ }^{4}$, and G. K. Parks \\ ${ }^{1}$ Centre d'Etude des Environnements Terrestre et Planétaires, 4 avenue de Neptune, 94107 Saint-Maur-des-Fossés Cedex, \\ France \\ ${ }^{2}$ Geophysical Research Division, Finnish Meteorological Institute, P.O. Box 503, 00101 Helsinki, Finland \\ ${ }^{3}$ Department of Physics and Astronomy, University of Leicester, University Road, Leicester, LE1 7RH, UK \\ ${ }^{4}$ Space Sciences Laboratory, University of California, Berkeley, CA 94720-7450, USA
}

Received: 13 December 2002 - Revised: 5 May 2003 - Accepted: 4 June 2003 - Published: 1 January 2004

\begin{abstract}
On 12 January 2000, during a northward IMF period, two successive conjunctions occur between the CUTLASS SuperDARN radar pair and the two satellites Ørsted and FAST. This situation is used to describe and model the electrodynamic of a nightside meso-scale arc associated with a convection shear. Three field-aligned current sheets, one upward and two downward on both sides, are observed.

Based on the measurements of the parallel currents and either the conductance or the electric field profile, a model of the ionospheric current closure is developed along each satellite orbit. This model is one-dimensional, in a first attempt and a two-dimensional model is tested for the Ørsted case. These models allow one to quantify the balance between electric field gradients and ionospheric conductance gradients in the closure of the field-aligned currents.

These radar and satellite data are also combined with images from Polar-UVI, allowing for a description of the time evolution of the arc between the two satellite passes. The arc is very dynamic, in spite of quiet solar wind conditions. Periodic enhancements of the convection and of electron precipitation associated with the arc are observed, probably associated with quasi-periodic injections of particles due to reconnection in the magnetotail. Also, a northward shift and a reorganisation of the precipitation pattern are observed, together with a southward shift of the convection shear.
\end{abstract}

Key words. Ionosphere (auroral ionosphere; electric fields and currents; particle precipitation) - Magnetospheric physics (magnetosphere-ionosphere interactions)

\section{Introduction}

Convection shears associated with multiple field-aligned current (FAC) sheets are a common feature of the nightside ionosphere. Gurnett and Frank (1973) have reported the existence of pairs of narrow, intense and oppositely directed electric

Correspondence to: A. Marchaudon

(aurelie.marchaudon@ cetp.ipsl.fr) field signatures associated with inverted-V electron precipitation. These pairs of opposite electric fields are responsible of ionospheric convection shears and of field-aligned currents due to the divergence of the ionospheric Pedersen currents. Later, Burch et al. (1976) and Huang et al. (1984) have studied the detailed structure of several convection shears in the nightside ionosphere. Burch et al. (1976) have shown that the inverted- $V$ electron precipitation was not exactly located at the associated convection reversal and that the electric field pattern was related to three intense field-aligned currents sheets, with an upward current at the centre and two downward return currents, one on its poleward side and one on its equatorward side. These parallel currents are closed by ionospheric Pedersen currents. Marklund (1984), in his auroral arc classification, has given them the name of "Birkeland current arcs". The basis of his classification is the fact that current continuity across the arc can be maintained either by polarization electric fields or by field-aligned currents. Over the past 30 years, electrodynamic studies have concerned either "polarization" dominated arcs (de la Beaujardière et al., 1977; Marklund et al., 1982, 1983; Wahlund and Opgenoorth, 1989; Janhunen et al., 2000) or "Birkeland current" dominated arcs (Burch et al., 1976; Aikio et al., 1993, 1995, 2002; Johnson et al., 1998). However, most of these arcs combine simultaneously strong perpendicular electric fields gradients and field-aligned currents.

During northward IMF periods, in a statistical analysis of nightside large-scale currents, Taguchi (1992) identified midnight sector triple-sheet (MTS) field-aligned current systems. He showed that the location and structure of the current system are controlled by the sign of the IMF $B_{y}$ component. In the Northern Hemisphere and for negative $B_{y}$, the current system occurs in the dawnside ionosphere, with an upward central current and downward currents on both sides. For a positive $B_{y}$, the current system occurs in the duskside and the sign of the currents is reversed. This study was later extended to the full DE-2 data set by Taguchi et al. (1994). These data allowed one to obtain statistical convection and electron precipitation patterns associated with the system of 


\section{SD FOV \& Oersted and FAST orbits -22:20 UT}

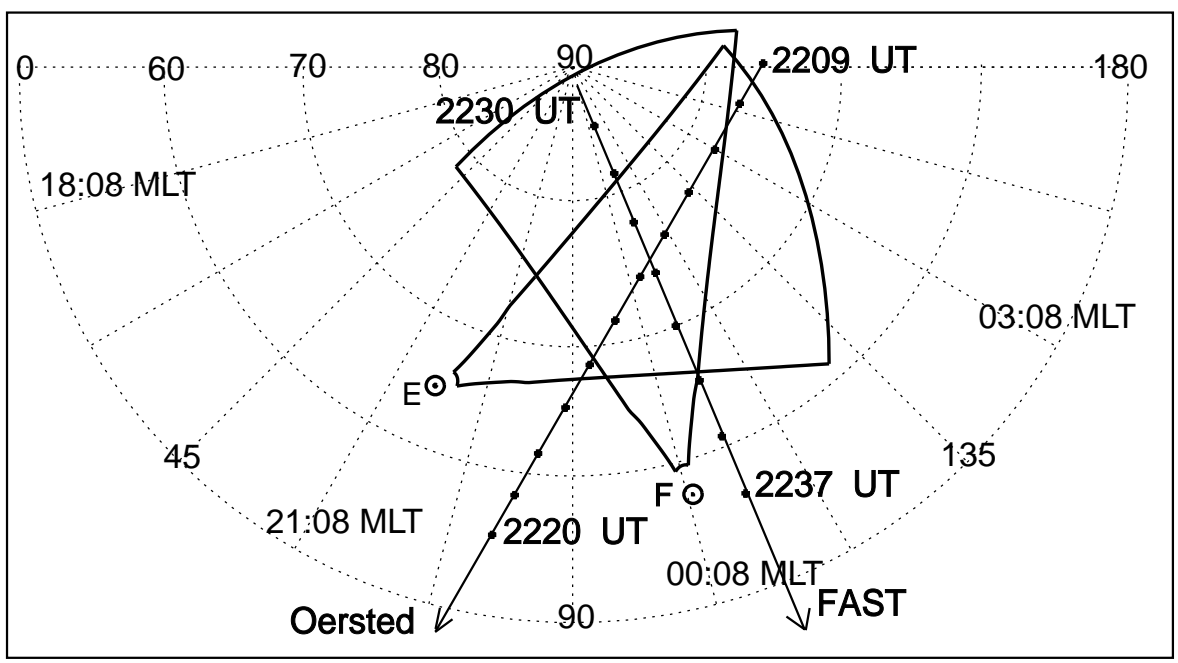

Fig. 1. Common field-of-view in magnetic coordinates of the CUTLASS Hankasalmi (F) and Pikkvibær (E) radars at 22:20 UT, with the FAST and Ørsted trajectories superimposed. three field-aligned currents. In the Northern Hemisphere for negative $B_{y}$, the convection consists of a velocity shear, with a westward plasma flow at the lower latitudes and an eastward plasma flow at the higher latitudes. For positive $B_{y}$, the flow directions are reversed. The electron precipitation disappears close to the convection reversal boundary and is always located on the low-latitude side of the plasma flow.

Different techniques have been used to model the horizontal and parallel currents system (see Untiedt and Baumjohann, 1993, for a review). The "method of characteristics" (Amm, 1995, 1997, 1998) has allowed Kosch et al. (2000) to model a vortex associated with an auroral arc. The horizontal and field-aligned currents and the ionospheric conductivity distributions are deduced over a $200 \mathrm{~km} \times 200 \mathrm{~km}$ region, based on ionospheric plasma velocities obtained with the STARE radar and magnetic field disturbances deduced from the Scandinavian Magnetometer Array (SMA). They showed that the plasma flow vortex was associated with an ionospheric region of diverging horizontal electric field, equivalent to a downward field-aligned current, and corresponding to a region of decreased conductivities, as expected for an anticlockwise convection. This was the first observation of a black aurora from the ground.

In this paper, we present a case study on 12 January 2000 of a convection shear during relatively quiet and steady IMF conditions characterised by a positive $B_{z}$ and a negative $B_{y}$. This shear is representative of the negative $B_{y}$ case of Taguchi et al. (1994). The data are obtained during a conjunction in the midnight sector between the Ørsted and FAST satellites, occurring over the common field of view of the CUTLASS pair of the SuperDARN radars. Ørsted crosses the common field of view of the radars between 22:10 and 22:16 UT and FAST 20 min later, between 22:30 and 22:36 UT, as shown on the schematic diagram of Fig. 1. The two satellite passes will be presented and modelled separately since they are separated by $20 \mathrm{~min}$. For the FAST pass, field-aligned currents deduced from magnetic field perturbations and ionospheric conductivities deduced from particle precipitation will be used to determine the ionospheric convection profile along the orbit, by using the condition of current continuity. For the Ørsted pass, using the fieldaligned currents deduced from Ørsted and ionospheric convection from SuperDARN radar data, the latitudinal profile of the ionospheric Pedersen conductance will be deduced, again from the condition of current continuity. A comparison between the results obtained in both cases will be made, in order to feature the similarities and differences due to time and space separation. Auroral images from the Polar-UVI instrument will be used to emphasize the time evolution of the arc between the Ørsted and FAST satellite passes.

\section{Instrumentation}

The solar wind plasma and IMF data are provided by the ACE satellite, and images of the nightside auroral oval are from the Ultraviolet Imager (UVI) on board the Polar satellite.

The ionospheric convection velocities are given by the Hankasalmi (in Finland) and Pikkvibær (in Iceland) CUTLASS radars. These radars belong to the SuperDARN chain of coherent HF radars, of which the main objective is to monitor the large-scale convection in the high-latitude ionosphere (Greenwald et al., 1995). Each radar measures the line-ofsight plasma flow velocity in the F-region of the ionosphere. The Hankasalmi and Pikkvibær radars share a common field of view, thus allowing one to obtain convection velocity vectors from the simultaneous measurement of two independent components. The SuperDARN radar beam is narrow, typically $3.3^{\circ}$ in azimuthal width. In the common mode, the radar beam scans successively 16 adjacent directions and is divided into 75 range bins of $45 \mathrm{~km}$ in length. Thus, the field of view of each radar is $3000 \mathrm{~km}$ in range and $53^{\circ}$ in azimuth. 
ACE IMF and solar wind data - 12/01/2000

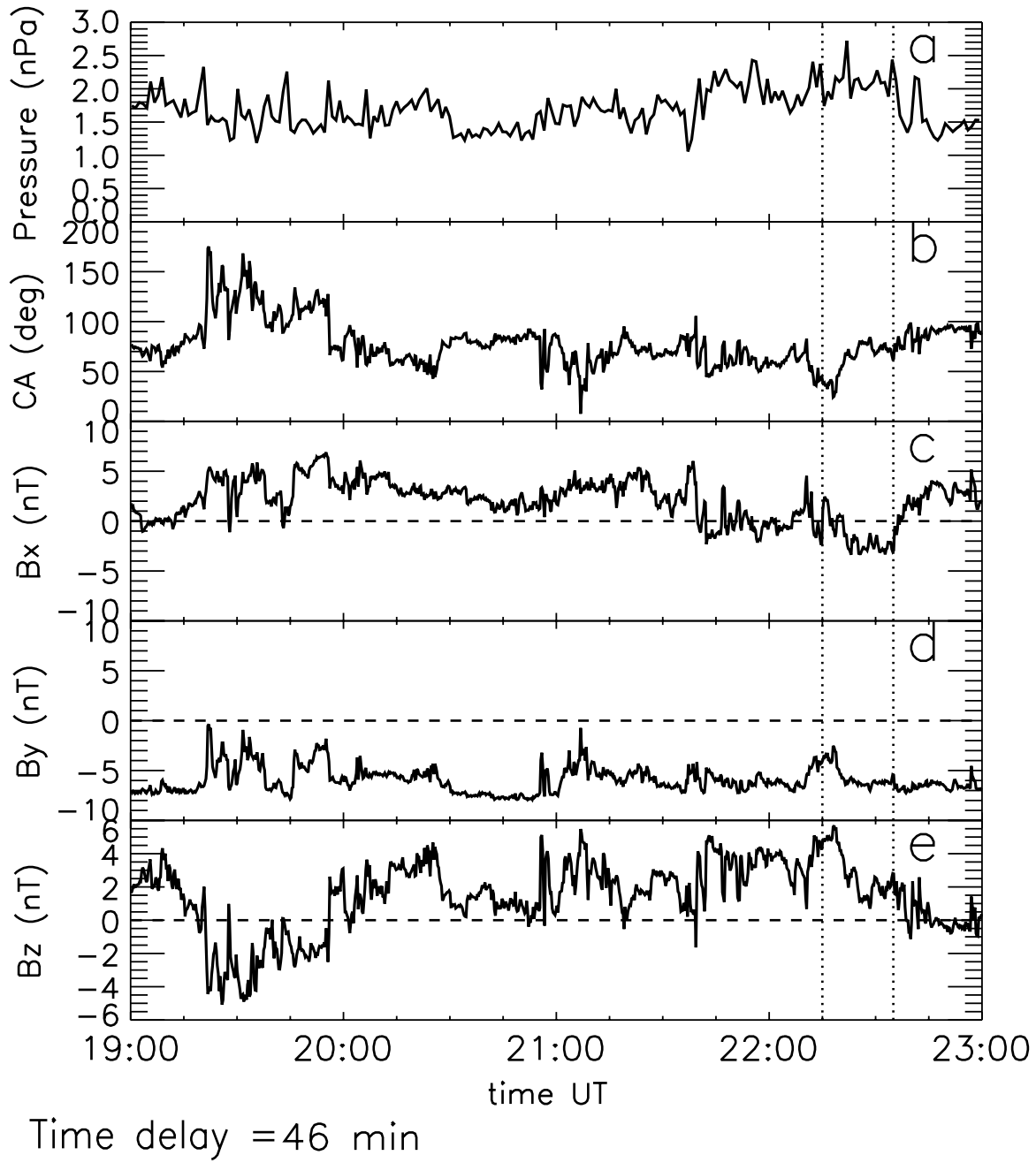

Fig. 2. Interplanetary magnetic field (IMF) and solar wind plasma data from the ACE spacecraft on 12 January 2000. Data are lagged by $46 \mathrm{~min}$, to take into account the delay from the spacecraft to the ionosphere. (a) Solar wind pressure. (b) IMF clock angle. Panels (c), (d) and (e) show, respectively, the IMF $B_{x}, B_{y}$ and $B_{z}$ components in GSM coordinates.
In the present study, the radars were operated in a high resolution mode, in which a full scan was completed in $35 \mathrm{~s}$.

During the first conjunction with the Ørsted satellite, fieldaligned currents are deduced from magnetic field measurements. Ørsted is a Danish satellite, with a quasi-polar orbit at about $600 \mathrm{~km}$ altitude. Its main objective is to perform a precise mapping of the Earth's magnetic field based on both a 3-axis and a scalar magnetometer. A detailed description of the satellite and its instrumentation are given by Stauning et al. (2001). During the second conjunction with the FAST satellite, we will use field-aligned currents, electric field and downgoing electron precipitation data. FAST is on a low-altitude, elliptical polar orbit and is designed to study small-scale structures of space plasma and acceleration mechanisms of particles (Carlson et al., 1998).

\section{General context}

Solar wind and IMF data from the ACE satellite are presented in Fig. 2 (GSM coordinates). The propagation de- lay between ACE and the dayside ionosphere is evaluated to about 46 min, by which the data in Fig. 2 have been lagged. The time of the Ørsted and FAST satellites' passes over the radars' field of view is indicated by the vertical dotted lines. The IMF $B_{z}$ remains positive for more than $2 \mathrm{~h}$ before the satellite passes. At the time of the satellites' passes, the average $B_{z}$ is $+4 \mathrm{nT}$ for Ørsted and $+2 \mathrm{nT}$ for FAST. The $B_{y}$ component remains negative and stable, with an average value of $-6 \mathrm{nT}$, and the $B_{x}$ component changes from positive to negative at the time of the Ørsted pass. Thus, a stable convection pattern is expected in the entire polar cap, characteristic of a strongly negative $B_{y}$ and a positive $B_{z}$. The average value of the IMF clock angle between 20:00 and 23:00 UT is $70^{\circ}$. Taguchi and Hoffman (1996) have used a limiting value of $70^{\circ}$ for the clock angle for the IMF $B_{y}$ control of the convection, which suggests that it is presently the case. Freeman et al. (1993) (for strong total IMF $(B=25 \mathrm{nT})$ ) and Senior et al. (2002) (for smaller total IMF) have shown that a clock angle of $70^{\circ}$ represent the transition at which dayside reconnection starts. Thus it is possible that during the period of 

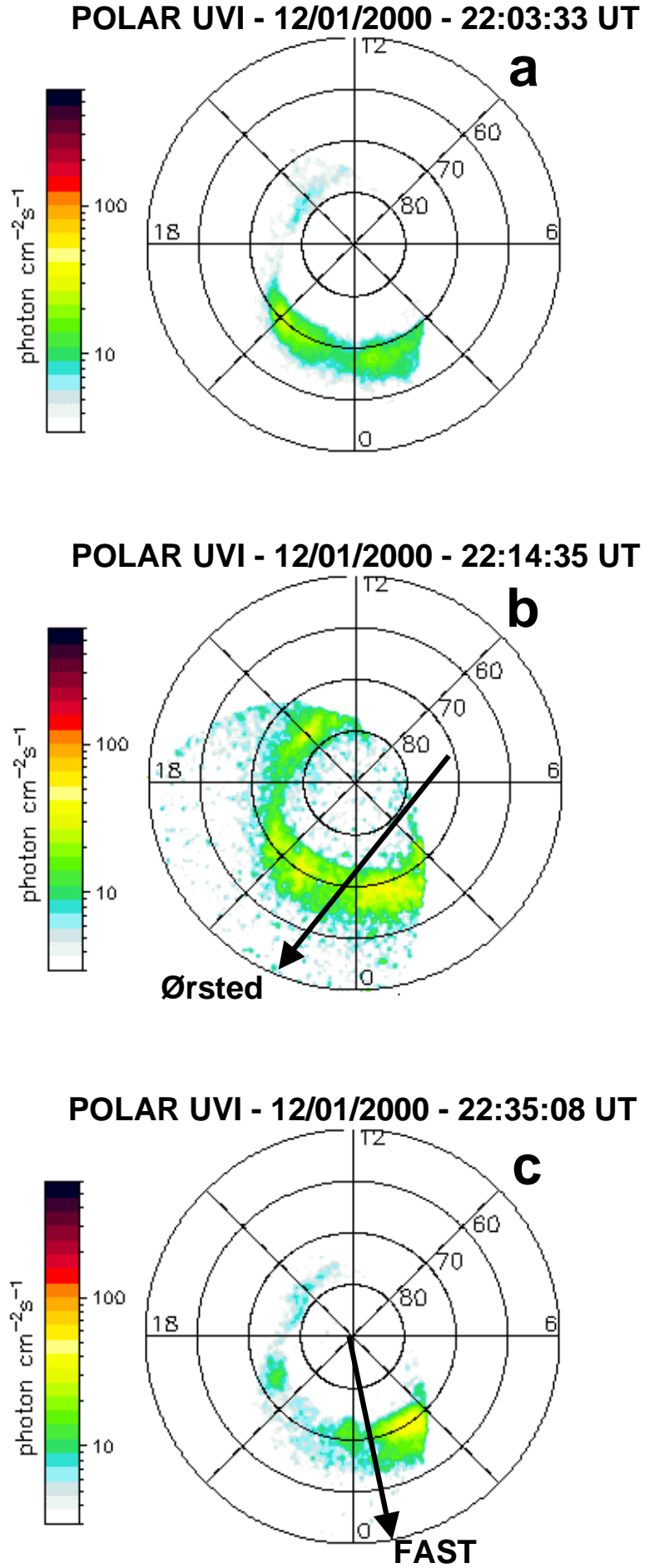

Fig. 3. Polar-UVI images in magnetic latitude-MLT coordinates. (a) $11 \mathrm{~min}$ before the Ørsted pass. (b) at the time of the Ørsted pass 22:14:35 UT, with the Ørsted trajectory superimposed. (c) at the time of the FAST pass 22:35:08 UT, with the FAST trajectory superimposed.

small clock angle (22:10-22:20 UT), no dayside reconnection exists, but that the polar cap continues to evacuate on the nightside, fossil open flux created by dayside reconnection.
Between 19:00 and 23:00 UT, the mean solar wind dynamic pressure is $1.7 \mathrm{nPa}$, increasing to $2.2 \mathrm{nPa}$ at the time of the satellites' passes. The solar wind and IMF conditions correspond to a quiet and fairly steady magnetosphere, mainly controlled by the negative $B_{y}$, a situation similar to the statistical studies of Taguchi (1992), Taguchi et al. (1994) and Taguchi and Hoffman (1996).

During the period of interest, Polar-UVI observations of the nightside auroral oval are available. The Polar-UVI images obtained $11 \mathrm{~min}$ before the Ørsted pass (Fig. 3a), at the time of the Ørsted pass (Fig. 3b) and at the time of the FAST pass (Fig. 3c) illustrate the spatial structure and the temporal variation of the nightside precipitation during the period and show that even if the magnetosphere is in a quiet configuration, the situation is, however, quite dynamic. Both satellite passes occur on the western edge of a precipitation zone covering the early morning sector. By comparing these 3 images, an apparent poleward motion of the auroral oval is observed in the midnight and early morning sector, especially between the first 2 images, which corresponds to the time interval 22:05-22:15 UT when the clock angle is smallest (Fig. 2a). This shrinking of the polar cap size is in agreement with the hypothesis of a decrease in the open flux in the polar cap. The 3 images also illustrate intensity variations of the precipitation pattern. The first and third images show a weak precipitation intensity while the second image shows an increase of the precipitation intensity. Moreover, examination of all the Polar images of the global time interval 22:00-23:00 UT indicates intensifications of the auroral precipitation about every $6 \mathrm{~min}$, which confirms the dynamical situation. A maximum of precipitation is observed just before the Ørsted pass and a minimum of precipitation is observed during the FAST pass. Auroral quasi-periodic intensifications under similar IMF conditions have been already observed by de la Beaujardière et al. (1994) and Senior et al. (2002).

\section{Data presentation}

\subsection{Ionospheric convection}

Figures $4 \mathrm{a}$ and $\mathrm{b}$ show the pattern of ionospheric convection deduced from the CUTLASS radars, in the midnight sector for the two periods of interest: 22:10-22:16 UT, corresponding to the Ørsted pass and 22:30-22:36 UT, corresponding to the FAST pass. The coordinates are AltitudeAdjusted Corrected GeoMagnetic (AACGM) (Baker and Wing, 1989) Magnetic Latitude (MLAT) and Magnetic Local Time (MLT). The maps have been averaged over 6 min in order to obtain more convection vectors. The projection along magnetic field lines of the Ørsted pass is superimposed on Fig. $4 \mathrm{a}$ and the projection of FAST pass on Fig. $4 \mathrm{~b}$.

During the first period, the convection is complex, with a partial clockwise vortex to the west, terminated to the east by a velocity shear, with eastward and slightly southward velocities at the higher latitudes and purely westward velocities at 


\section{SUPERDARN VELOCITY MAP - Thykkvibaer / Hankasalmi}
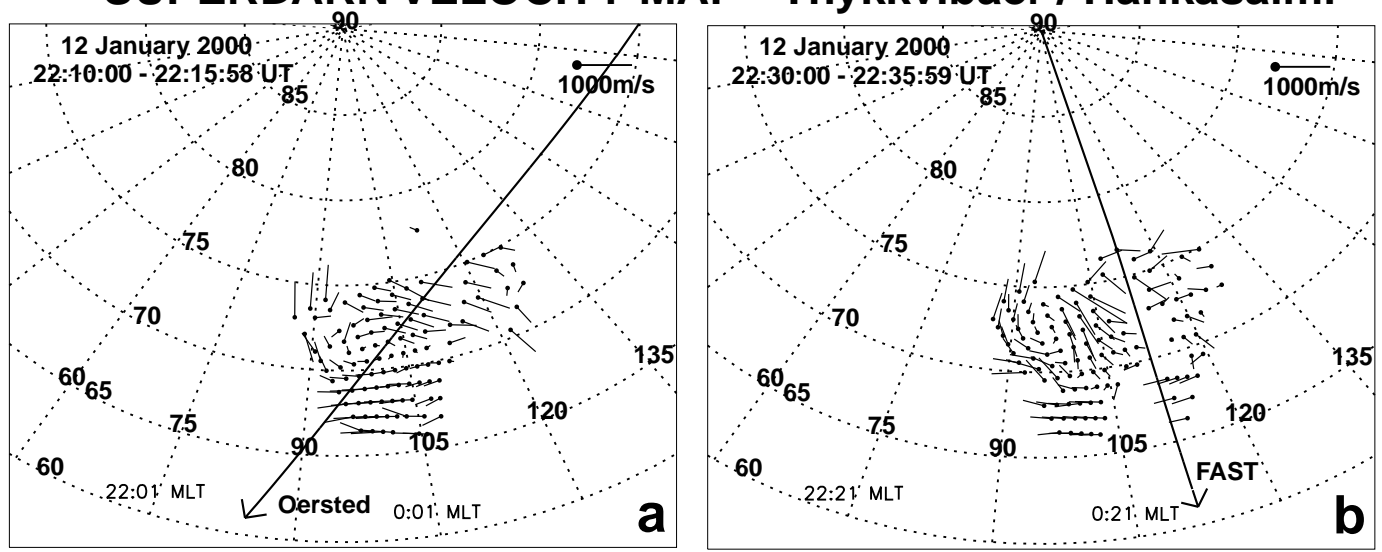

Fig. 4. Average (over $6 \mathrm{~min}$ ) convection velocity maps of the CUTLASS radars in AACGM coordinates. (a) at the time of the Ørsted pass 22:10-22:16 UT, with the Ørsted trajectory superimposed. (b) at the time of the FAST pass 22:30-22:36 UT, with the FAST trajectory superimposed.

the lower latitudes. The dimensions of the structure are fairly large, with a $750 \mathrm{~km}$ extension in longitude and $400 \mathrm{~km}$ in latitude. The centre of the shear is at about $71.5^{\circ}$ MLAT. The convection velocity is of the order of $600 \mathrm{~m} \mathrm{~s}^{-1}$ on the edge of the structure. This semi-opened convection vortex encircles the early morning precipitation zone observed in Fig. $3 b$. The Ørsted trajectory superimposed on this map shows that Ørsted crosses the southwestern part of the convection vortex.

At the time of the FAST pass 20 min later, the convection (Fig. 4b) has changed significantly. A clockwise rotation of the vortical structure has occurred in the western part of the map. The velocities have the same amplitude, except for a small zone centred around $72^{\circ}$ MLAT and are composed of larger and predominantly southward velocities. This southward flow indicates that the western part of the shear has evolved into an autonomous vortex, encircling the precipitation spot centred at $70^{\circ}$ MLAT and 00:00 MLT in Fig. 3c. The trajectory superimposed on Fig. 4b shows that FAST crosses the eastern part of the convection pattern. In this region, almost the same directions of velocity are observed as at the time of the Ørsted pass. Around $75^{\circ}$ MLAT, predominantly eastward velocities are observed as well as westward velocities below $68.5^{\circ}$ MLAT. However, because data points are missing in the SuperDARN convection map along the FAST orbit, the position of the velocity shear is not well defined. The north-south component of the DC electric field measured by FAST (Fig. 5a) and corresponding to the eastwest component of the convection, indicates a convection reversal from eastward above $68.5^{\circ}$ MLAT to westward below $68.5^{\circ}$ MLAT. Thus, the position of the convection shear has evolved between the two satellites passes and is shifted southward at the time of the FAST pass. This observation confirms the evolution of the convection pattern. Unfortunately, the east-west component of the DC electric field at FAST, which could indicate a southward component of the convection flow, is not available.

Differences between SuperDARN and FAST data can be explained by the spatial and temporal smoothing of the SuperDARN convection maps. The electric field observed by FAST shows small-scale structures, in particular, a significant reversal of the electric field gradient at $71^{\circ}$ MLAT, where the electric field becomes almost zero. Notice also the strong positive electric field around $73^{\circ}$ MLAT, the amplitude of which will be discussed later. These two features (gradient reversal at $71^{\circ}$ MLAT and peak electric field at $73^{\circ}$ MLAT) are essential because, as we will show later, they are linked to field-aligned currents.

\subsection{Field-aligned currents}

The field-aligned currents associated with the convection shear are deduced from measurements made by the Ørsted satellite during the first period, and by the FAST satellite during the second period. The two satellites are moving equatorward in the nightside. The magnetic perturbation $\delta \boldsymbol{B}$ is obtained by subtracting from the magnetic measurements the IGRF model of the internal magnetic field. The field-aligned currents $J_{\|}$is deduced from Ampere's law

$J_{\|}=\frac{1}{\mu_{o}} \nabla \times \delta \boldsymbol{B}$,

assuming current sheets, the orientation of which is parallel to $\delta \boldsymbol{B}$.

The magnetic perturbation at the Ørsted orbit and the associated field-aligned currents are shown in Fig. 6. The southward component (panel (a) is relatively weak, with variations less than $100 \mathrm{nT}$ and the eastward component (panel (b) has a sawtooth-like shape, with variations up to $250 \mathrm{nT}$. Large-scale variations reveal the existence of three current sheets. Between 22:13:00 and 22:14:30 UT (75.55 to $72.2^{\circ}$ MLAT), a smooth decrease in the eastward perturbation is observed, with an accentuation of the decrease at 
FAST : 12 january 2000

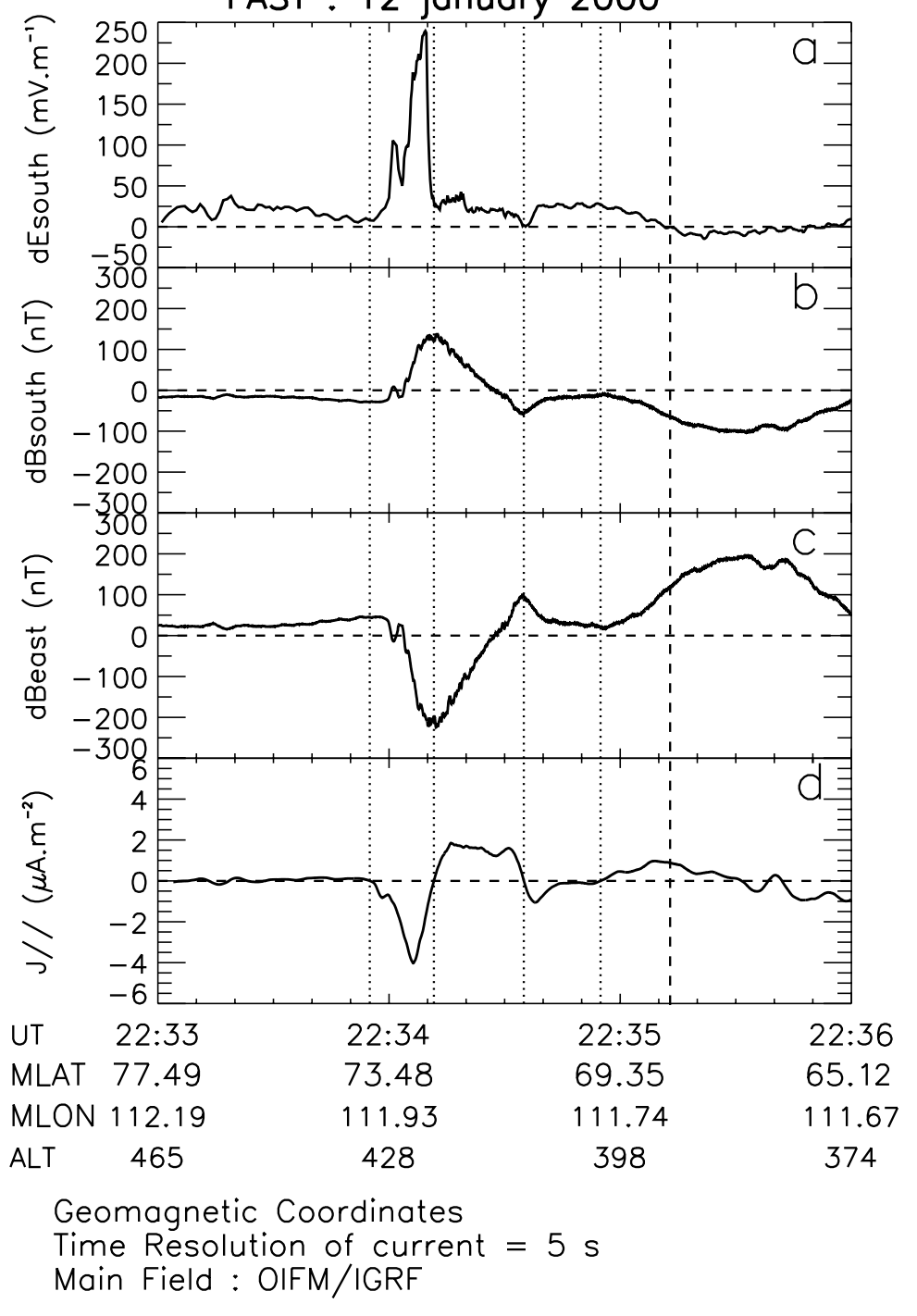

Fig. 5. Electric and magnetic fields and field-aligned currents measured by FAST. (a) North-south component of the convection electric field. (b) and (c) North-south and east-west components of the magnetic perturbations. (d) Field-aligned currents intensity deduced from the magnetic perturbations, averaged over $5 \mathrm{~s}$. Dotted lines delineate the three parallel current sheets and the dashed line indicates the velocity shear. low-latitudes which could be associated with a small-scale structure superimposed on the global structure. Then, between $22: 14: 30$ and $22: 16: 30 \mathrm{UT}\left(72.2^{\circ}\right.$ to $\left.66.3^{\circ} \mathrm{MLAT}\right)$, in spite of large perturbations, a general positive increase in this component is observed. Finally, at lower latitudes, between $22: 16: 30$ and 22:17:00 UT (66.3 ${ }^{\circ}$ to $64.7^{\circ}$ MLAT), a decrease in the perturbation (not shown in Fig. 6) is observed. These three perturbations agree with the statistical model of Iijima and Potemra (1976) around midnight which includes three (downward-upward-downward) large-scale currents, namely the morningside region-1, the eveningside regions- 1 and -2 currents. The latitudinal extent of these currents is large, probably related to the positive IMF $B_{z}$. Smaller scale perturbations with a larger amplitude are observed between $22: 14: 13$ and 22:15:12 UT, at the interface between the morningside and the eveningside regions1. Three intense current sheets deduced from these perturbations are represented in Fig. 6c, including from north to south: a downward current, an upward current centred on the velocity shear and a downward current. These currents will be called, respectively, in the following sections: $N_{H}$ current for the High-latitude Negative (downward) current, $P$ current for the Positive (upward) central current and $N_{L}$ current for the Low-latitude Negative current. These currents have quite large intensities, up to $5 \mu \mathrm{A} \mathrm{m}^{-2}$ and their latitudinal width is, respectively, $65 \mathrm{~km}, 140 \mathrm{~km}$ and $75 \mathrm{~km}$, for $N_{H}, P$ and $N_{L}$. The $P$ current corresponds to the clockwise circulation of the convection semi-vortex, and the $N_{H}$ and $N_{L}$ currents correspond to the return currents of the convectionprecipitation-upward current structure. The sign of these currents agrees with the MTS current system of Taguchi (1992), for negative $B_{y}$.

During the FAST pass, the eastward component of the magnetic perturbation is qualitatively similar to that obtained on board Ørsted, although the amplitudes are different (Fig. 5b and c). In particular, the three large-scale variations corresponding to the morningside region-1, the eveningside regions- 1 and -2 currents are also observed. The latitudinal 
Oersted : 12 january 2000

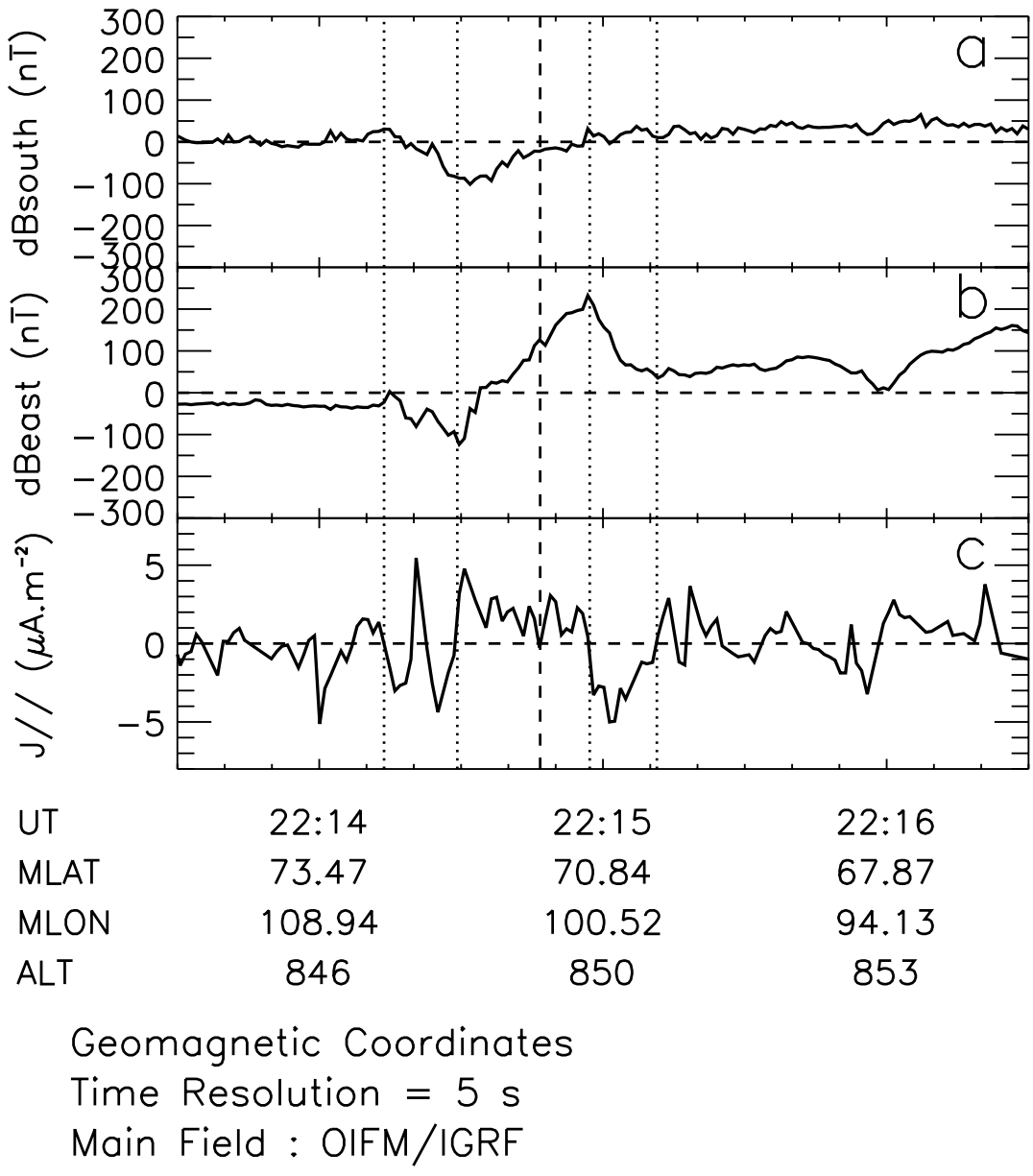

Fig. 6. Magnetic field and fieldaligned currents measured by Ørsted. (a) North-south component. (b) Eastwest component. (c) Field-aligned currents intensity averaged over $5 \mathrm{~s}$. Dotted lines delineate the three parallel current sheets and the dashed line indicates the velocity shear.
Table 1. Angle between the directions of the magnetic East and of the 3 current sheets associated with the arc, along the Ørsted and FAST orbits. The angles are positive clockwise

\begin{tabular}{lcc}
\hline & Ørsted pass & FAST pass \\
\hline$N_{H}$ current & $+68.0^{\circ}$ & $-7.5^{\circ}$ \\
$P$ current & $+39.5^{\circ}$ & $-9.0^{\circ}$ \\
$N_{L}$ current & $+15.5^{\circ}$ & $-9.5^{\circ}$ \\
\hline
\end{tabular}

structure of the smaller scale parallel current is also similar to the Ørsted case, with three current sheets embedded in region-1. These currents have the same sign for the two satellites passes. In the FAST case, the latitudinal width is, respectively, $125 \mathrm{~km}, 175 \mathrm{~km}$ and $160 \mathrm{~km}$ for $N_{H}, P$ and $N_{L}$, which is slightly larger than the widths calculated along the Ørsted orbit. Table 1 shows the angle (positive anticlockwise) between the magnetic isolatitude and the direction of the current sheet. The current sheets are nearly parallel to magnetic isolatitude lines at FAST, while they rotate along the Ørsted orbit. This important difference will be interpreted in the Discussion section. The amplitude of the $P$ and $N_{L}$ currents is smaller along the FAST orbit (Fig. 5d).

\subsection{Particle precipitation}

Figure 7a displays the electron precipitation measured by FAST. The precipitation pattern is highly structured with a strong precipitation above $71.3^{\circ}$ MLAT, from north to south, a gap associated with the $N_{L}$ current, and a weaker but more extended precipitation zone below $70^{\circ}$ MLAT. This precipitation pattern is often observed in association with a convection reversal, as, for instance, by Gurnett and Franck (1973). From the electron precipitation pattern, we determine the Pedersen and Hall conductances (height-integrated conductivities), using Hardy's model (Hardy et al., 1987):

$$
\begin{aligned}
& \Sigma_{P}=\frac{40\langle\boldsymbol{E}\rangle}{16+\langle\boldsymbol{E}\rangle^{2}} \sqrt{\Phi} \\
& \Sigma_{H}=0.45\langle\boldsymbol{E}\rangle^{5 / 8} \Sigma_{P},
\end{aligned}
$$

where $\Sigma_{P}$ et $\Sigma_{H}$ are the ionospheric Pedersen and Hall conductances, respectively, $\langle\boldsymbol{E}\rangle$ is the electron mean energy in 
FAST: 12 January 2000

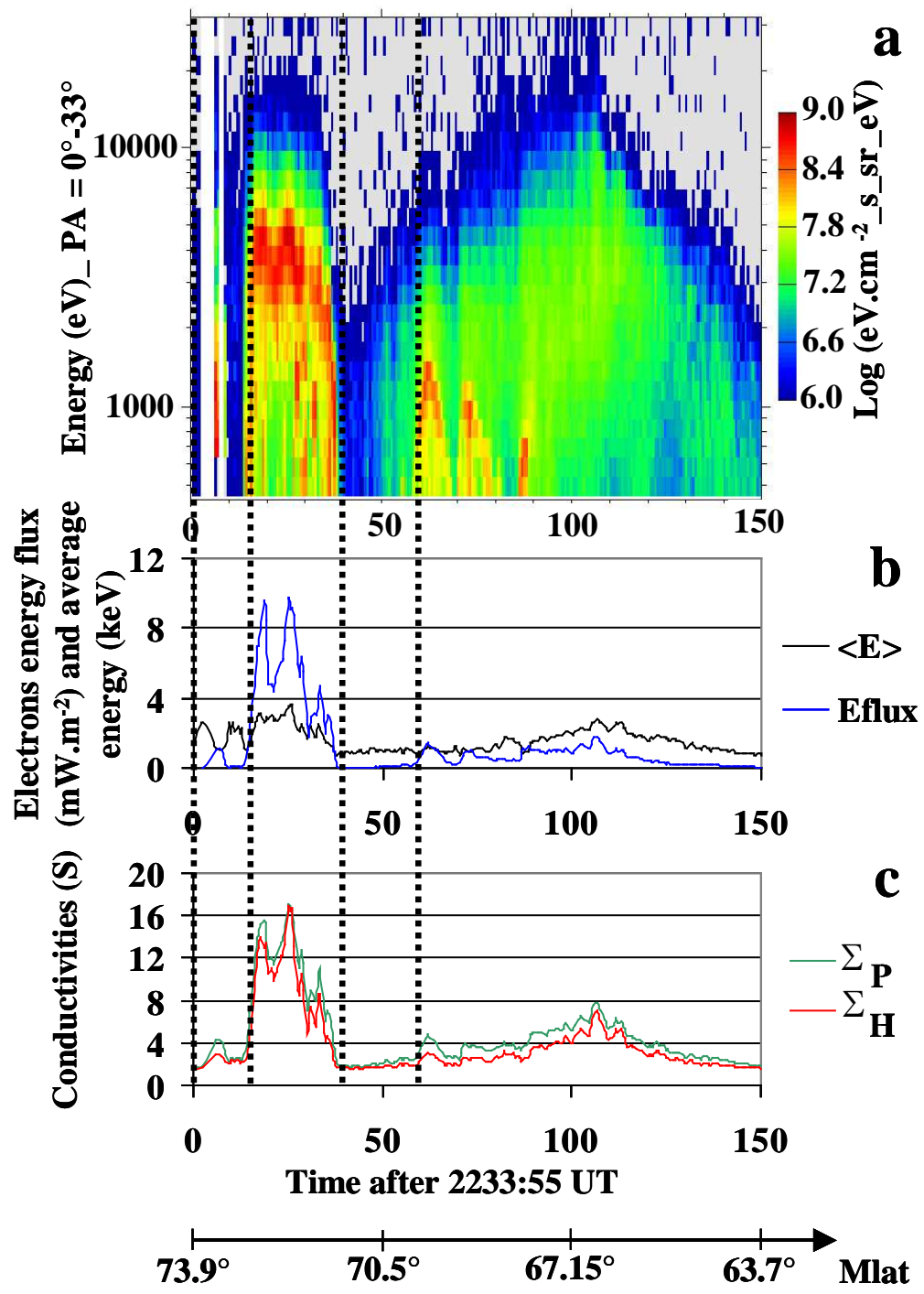

Fig. 7. Electron precipitation measured by FAST, between 22:33:55 and 22:36:25 UT. (a) Energy-flux diagram of the downgoing electrons, for the range $400 \mathrm{eV}-30 \mathrm{keV}$. (b) Energy flux and average energy. (c) Pedersen and Hall ionospheric conductances. The position of the three field-aligned current sheets deduced from FAST magnetic perturbations is superimposed (dotted lines).
$\mathrm{keV}$ with a low-energy cutoff at $500 \mathrm{eV}$, and $\Phi$ is the electrons energy flux in $\mathrm{mW} \mathrm{m}^{-2}$ (Fig. 7b). A basic ionospheric conductance is added to the Pedersen and Hall conductances, in order to take into account the faint solar ionisation. For the zenith angle and the MLT prevailing during the observations, a value of $1.5 \mathrm{~S}$ is chosen (Senior, 1991). The resulting conductance profiles are shown in Fig. 7c. The conductances are large in the electron precipitation regions, reaching up to $15 \mathrm{~S}$. The Hall and Pedersen conductance profiles are similar. Strong gradients occur on the edges of the high-latitude precipitation region.

\section{Simulation}

Based on this detailed data set, including convection velocities, conductances and parallel currents, models can be built, based on the current continuity equation:

$J_{\|}=-\Sigma_{P}\left(\nabla_{\perp} \cdot \boldsymbol{E}_{\perp}\right)-\boldsymbol{E}_{\perp} \cdot \nabla_{\perp} \Sigma_{P}+\left(\boldsymbol{b} \times \boldsymbol{E}_{\perp}\right) \cdot \nabla_{\perp} \Sigma_{H},(4)$

where $\boldsymbol{b}$ is the unit vector parallel to the Earth's magnetic field. In this expression, the first term on the right-hand side represents the contribution to the parallel current density of the ionospheric electric field gradients, which can be deduced from the convection velocity. The second and third terms represent the contribution of the conductance gradients deduced from particle precipitation. Because satellite measurements are made only along the orbit, they do not provide sufficient inputs for a full two-dimensional model (2-D).

\subsection{One-dimensional model}

In a first step, we develop a one-dimensional (1-D) model. Relation (4) taking a simple form when longitudinal uniformity can be assumed. In a cartesian local reference frame, 
FAST : 12 January 2000

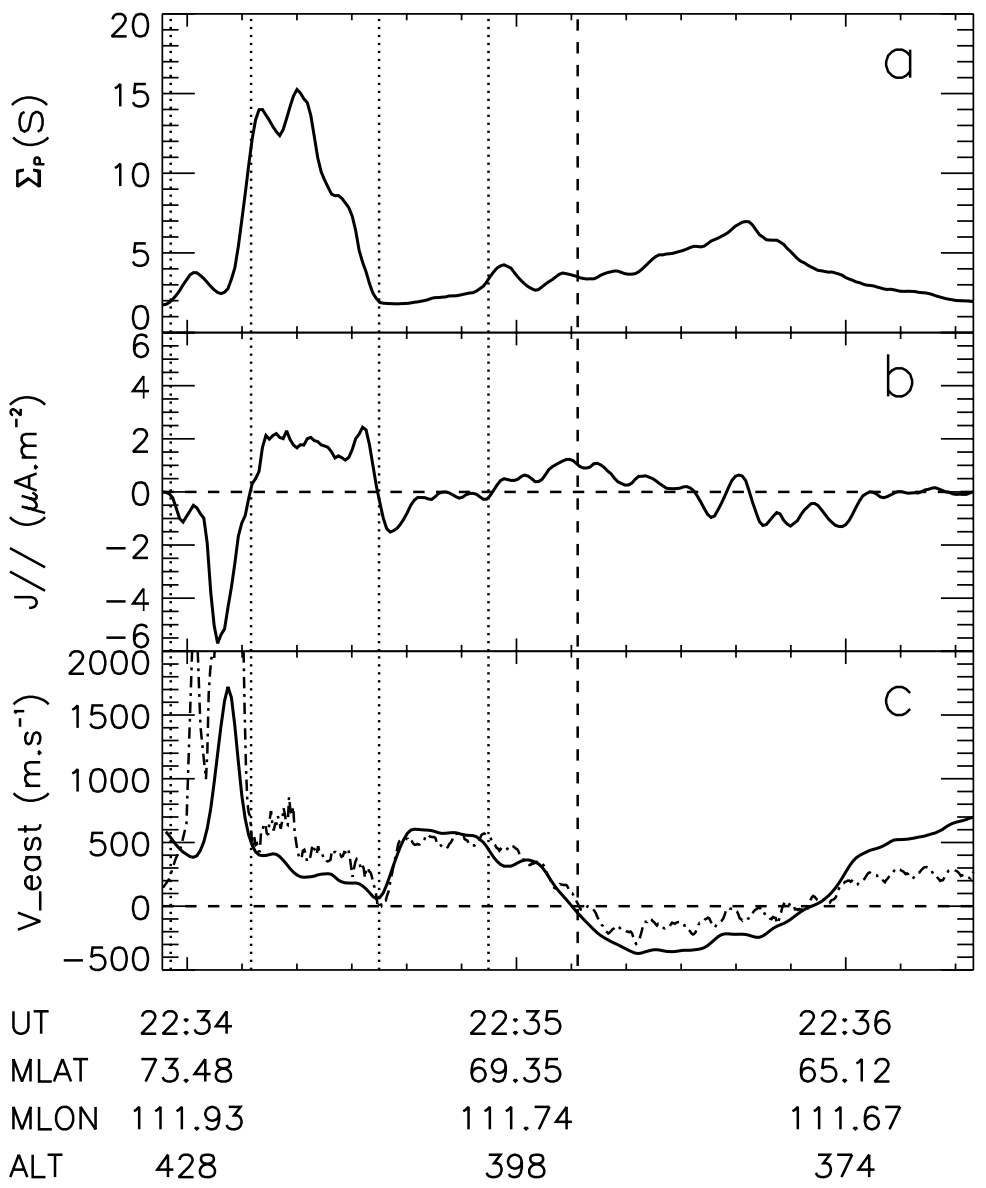

Fig. 8. Inputs (a and b) and result (c) parameters of the 1-D simulation along the FAST orbit. (a) Ionospheric Pedersen conductance. (b) Field-aligned current measured by FAST. (c) Modelled (solid) and experimental (dotted line) east-west convection velocity. Dotted lines delineate the three parallel current sheets and the dashed line indicates the velocity shear. where $x$ and $y$ represent the eastward and northward directions, and assuming a vertical magnetic field, relation (4) becomes:

$$
\begin{aligned}
& \frac{d V_{x}(y)}{d y}+\frac{1}{\Sigma_{P}(y)} \frac{d \Sigma_{P}(y)}{d y} V_{x}(y) \\
& =\frac{J_{\|}}{B \Sigma_{P}(y)}+\frac{1}{\Sigma_{P}(y)} \frac{d \Sigma_{H}(y)}{d y} V_{y}(y) .
\end{aligned}
$$

A usual simplification is to assume a constant Hall-toPedersen ratio of 1 , well supported by the conductance profiles along the FAST orbit (Fig. 7c)

$$
\frac{d V_{x}(y)}{d y}+\frac{1}{\Sigma_{P}(y)} \frac{d \Sigma_{P}(y)}{d y}\left[V_{x}(y)-V_{y}(y)\right]=\frac{J_{\perp}}{B \Sigma_{P}(y)} .(6)
$$

This equation can be regarded either as an algebraic equation giving the parallel current density or as a differential equation which governs the latitudinal profile of either the longitudinal component of the convection velocity or the Pedersen conductance, depending upon the parameters which are measured. In the latter cases, additional information on the $V_{y}$ component is necessary. We use relation (6) successively along the FAST and Ørsted orbits, in order to model the unknown (or less precisely measured) parameters.

\subsubsection{Simulation along the FAST orbit}

Along the FAST orbit, field-aligned currents have been measured and ionospheric conductances have been deduced from particle precipitation. On the other hand, as mentioned earlier, the east-west component of the DC electric field (giving the north-south component of the convection velocity) is not available on board the FAST satellite. Although SuperDARN convection vectors are very scarce in the region of the FAST trajectory, vectors at the east of the orbit, between $65^{\circ}$ and $75^{\circ}$ MLAT (Fig. 4b), are along the magnetic isolatitude lines. Consequently, we have chosen $V_{y}(y)=0$. Using field-aligned currents and conductances as inputs, relation (6) is integrated on the full latitudinal interval from $64^{\circ}$ to $74^{\circ}$ MLAT, with the fourth-order Runge-Kutta algorithm, in order to deduce the longitudinal component of the convection velocity. An initial value of the longitudinal component of the convection of $600 \mathrm{~m} \mathrm{~s}^{-1}$ is used at $74^{\circ}$ MLAT, in agreement with the experimental values of convection measured by FAST and SuperDARN. The inputs of Eq. (6) (Pedersen conductance and field-aligned currents) and the resulting longitudinal component of the convection velocity are shown in Fig. 8 (solid lines). This velocity profile can be compared ei- 


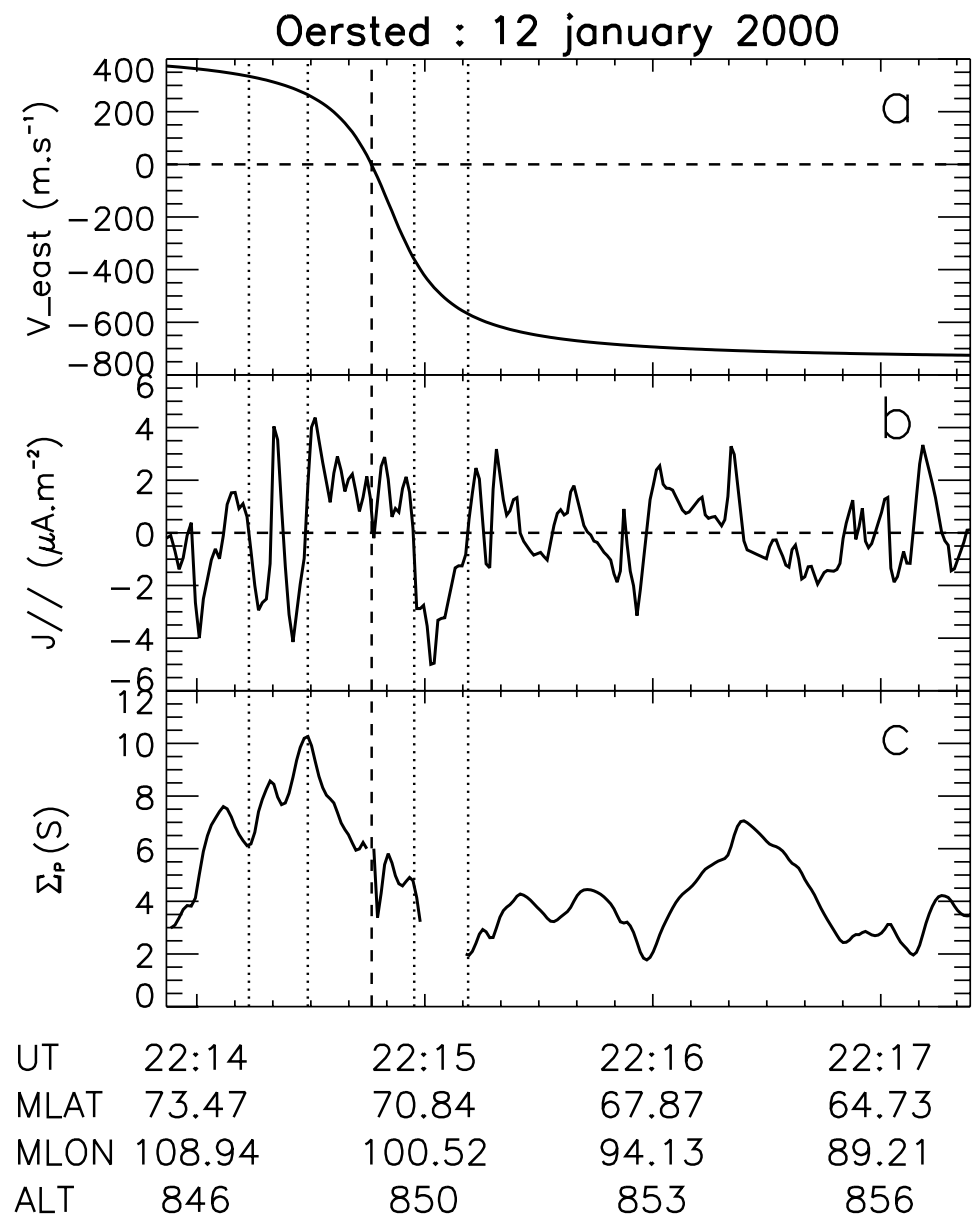

Fig. 9. Inputs (a and b) and result (c) parameters of the 1-D simulation along the Ørsted orbit. (a) East-west convection profile deduced from the $\mathrm{Su}$ perDARN convection map. (b) Fieldaligned current measured by Ørsted. (c) Modelled ionospheric Pedersen conductance. Dotted lines delineate the three parallel current sheets and the dashed line indicates the velocity shear.

ther with the east-west component of the convection velocity deduced from the north-south component of the electric field measured by FAST (dash-dotted line in Fig. 8) or with the SuperDARN measurements. Between $64^{\circ}$ and $74^{\circ}$ MLAT, the modelled and experimental data are very similar in position and amplitude, except for the discrepancy in amplitude observed above $72.5^{\circ}$ MLAT. The large velocity peak centred at $72.9^{\circ}$ MLAT is observed both in modelled and experimental data, but the amplitudes are very different. The reason for the difference between experimental and modelled amplitude of the electric peak will be explained in the Discussion section.

\subsubsection{Simulation along the Ørsted orbit}

Along the Ørsted orbit, field-aligned currents, convection velocity (and consequently convection electric field) are known. Using these inputs, relation (6) can be integrated, in order to deduce the Pedersen conductance. The inputs and the resulting latitudinal component of the Pedersen conductance along the Ørsted orbit are presented in Fig. 9. In a preliminary step, the experimental convection map (Fig. 4a) has been fitted to an analytic model. In this model, the east-west component of the ionospheric convection velocity $V_{x}(y)$ along the Ørsted orbit is characterised by a velocity shear at 71.5 MLAT (Fig. 9a), and the north-south component of the convection velocity is taken at $V_{y}(y)=-V_{x}(y) / 2$ above the velocity shear and $V_{y}(y)=0$ below. As a test of the role played by the north-south component of the velocity, we have also integrated Eq. (6) with a purely longitudinal velocity $\left(V_{y}(y)=0\right.$ everywhere). The result shows no significant difference in the conductance profile. At the velocity shear, relation (6) simplifies and the Pedersen conductance is obtained directly from the parallel current and the velocity gradient, which provides an initial value for integration from this point, independently on both sides of the convection reversal. The conductance at the velocity shear is $6 S$, a realistic value, since the discontinuity is situated on the low-latitude side of both the $P$ current and the electron precipitation and thus, corresponds to the lower side of the conductance gradient. We have chosen not to model the Pedersen conductivity in the region of the $N_{L}$ current, in order to avoid negative values. The integration starts again at $70.3^{\circ}$ MLAT, beyond the $N_{L}$ current. Poleward of the velocity shear, three peaks of conductance, with intensities decreasing from 10.5 to $8 \mathrm{~S}$, are obtained (Fig. 9c). Equatorward of the velocity shear, the Pedersen conductance is smaller, but three small amplitude peaks are obtained, with a maximum conductance of about 


\section{Oersted: 12 January 2000}
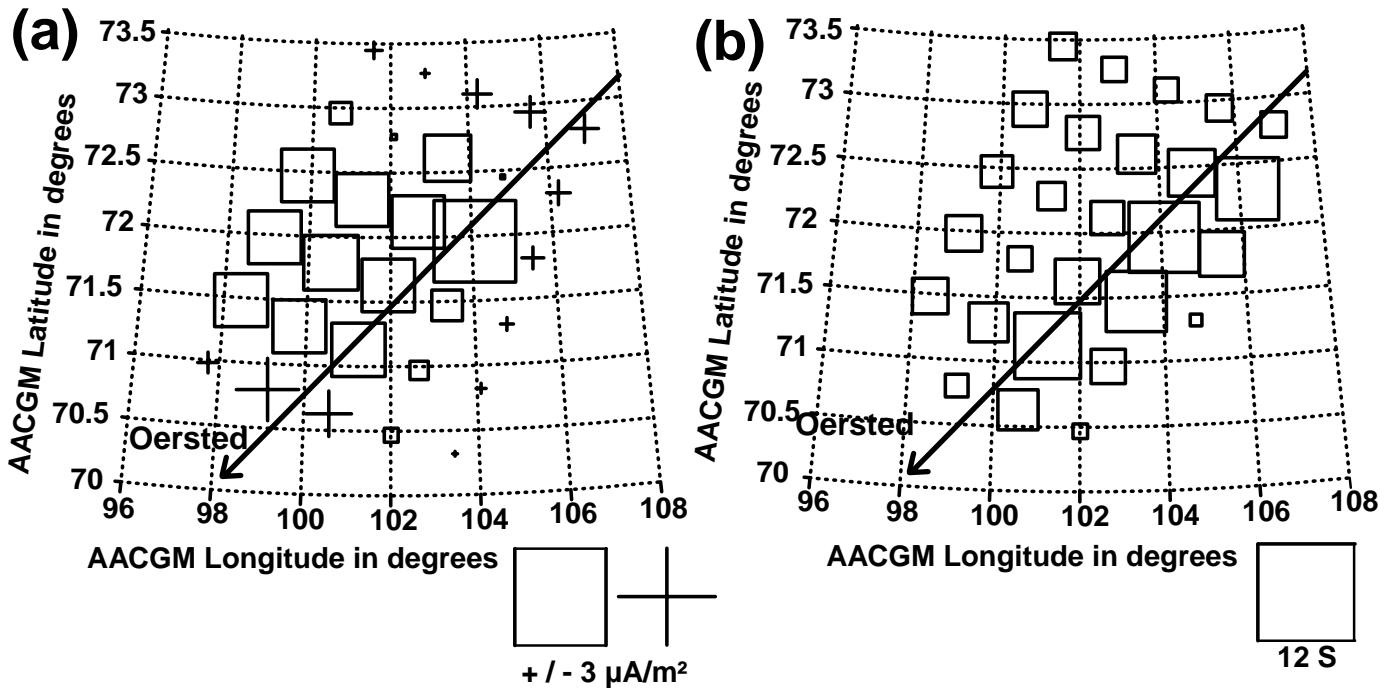

Fig. 10. Two-dimensional distributions in AACGM coordinates (a) of the field-aligned current deduced from the Ørsted measurements and (b) of the modelled Pedersen conductance, assuming a Hall-to-Pedersen conductance ratio equal to 1. The parameters are plotted in the form of symbols, of which the size is proportional to the intensity of the parameter. Squares and crosses represent, respectively, the upward currents and downward currents. The projection of the Ørsted orbit is also shown.

$7 \mathrm{~S}$, probably associated with the eveningside region-2 of a large-scale currents. The fact that we could not model the conductance in the region of the $N_{L}$ current shows that the 1-D model is not well adapted for the Ørsted orbit. However, in the regions where the conductance can be modelled, similarities between the experimental profile of Pedersen conductance along the FAST orbit and the modelled conductance along the Ørsted orbit are observed, although the time and longitude of FAST and Ørsted are different. Both poleward and equatorward of the shear, the number and the relative amplitude of the peaks are the same. A small global latitudinal shift, of the order of $0.2^{\circ}$ between the two profiles, is observed.

\subsection{Two-dimensional model along the Ørsted orbit}

Since along the Ørsted orbit the 1-D model is not successful in providing a full latitudinal profile of the Pedersen conductance, we have tested a 2-D model also based on the current continuity equation, obtained from the "FAC-based method of characteristics" developed by Amm (2002). This method allows one to calculate the distribution of ionospheric conductances and actual currents from the two-dimensional profiles of ionospheric electric field and of field-aligned currents mapped to the ionosphere, with the Hall-to-Pedersen conductance ratio $\alpha$ being given. The $\alpha$ ratio is taken to be 1 (a realistic value for low magnetic activity; see also the ratio of FAST conductances modelled in Fig. 7). The electric field pattern responsible of the convection vortex is given by the SuperDARN radars. Note that the centre of the convection vortex is located at $72.3^{\circ}$ MLAT and $101.3^{\circ}$ Magnetic Longitude (MLON), at a slightly higher latitude than the shear ob- served along the Ørsted orbit. However, because Ørsted provides FAC only along its orbit, it is necessary to extrapolate Ørsted data to a 2-D FAC distribution. The field-aligned current at each grid point is given by the mean of the FAC at the points of the Ørsted orbit situated at the same distance from the centre of the FAC. In order to have continuity of the current, the mean is weighted by the inverse distance between the data point and the Ørsted point. Before this process, the Ørsted data have been averaged to a scale of $\sim 50 \mathrm{~km}$, corresponding to the spatial resolution of SuperDARN. For the grid points at a radial distance smaller than the distance between the Ørsted orbit and the centre of the FAC distribution, the attributed value of FAC is equal to $+1.65 \mu \mathrm{A} \mathrm{m}^{-2}$. This value corresponds to the $50 \mathrm{~km}$ average of the closest portion of the Ørsted path from the vortex centre. The distribution of the FAC is plotted in AACGM coordinates in Fig. 10a. The FAC configuration shows a circular central positive current (the $P$ current) surrounded on its northward, southward and eastward sides by a downward current (corresponding to the $N_{L}$ and $N_{H}$ currents) with a smaller amplitude around $-0.6 \mu \mathrm{A} \mathrm{m}^{-2}$. Note that larger values of the currents are observed very close to the Ørsted orbit. Because the 2-D modelling is expected to provide satisfactory results only in the vicinity of the Ørsted orbit where field-aligned currents are true measurements, we have chosen to show only the central and eastern part of the current distribution in Fig. 10a. Figure 10b shows over the same area the calculated Pedersen conductance. The conductance shows a maximum reaching up to $8 \mathrm{~S}$, overlapping the $P$ current sheet and is elongated from southwest to northeast. The conductance decreases significantly to very small values in the downward FAC regions $\left(N_{L}\right.$ and $\left.N_{H}\right)$. The Ørsted satellite crosses this elongated 
maximum in its larger dimension. At the centre of the modelled positive current, the conductance decreases to about $3 \mathrm{~S}$. This surprising result will be explained in the next section.

\section{Discussion}

6.1 FAST results and the validity of the one-dimensional model

A strong similarity is observed between the experimental and the 1-D modelled profiles of the east-west convection (Fig. 8c). The forms of the peak around $72.9^{\circ}$ MLAT, the gradient reversal at $71^{\circ}$ MLAT and the convection shear at 68.5 MLAT are well reproduced by the simulation with only a small latitudinal shift of the convection shear. The amplitudes are also similar, except for the peak at $72.9^{\circ}$ MLAT, where the experimental peak is three times larger than modelled. Clearly, the amplitude is not realistic, suggesting a non-standard behaviour of the electric antenna in the $N_{H}$ region.

The $N_{H}$ current of the triple parallel currents' structure (Fig. 8b) is very intense, and its maximum is associated with a strong electric field gradient. This result confirms earlier studies, in which intense electric fields are observed in the downward FAC region adjacent to a brightening arc (Opgenoorth et al., 1990; Aikio et al., 1993, 2002). However, in our case, the latitudinal extent of the global arc, of the $N_{H}$ downward FAC, and of the associated electric field, are considerably larger. Moreover, a strong conductance gradient at the polar side of the precipitation and opposite to the electric field gradient occurs just at the boundary between the $N_{H}$ and the $P$ currents. The $P$ parallel current is associated with the conductance gradients related to the equatorward side of the electron precipitation. In the $P$ current, the electric field gradient is weak. At the boundary between the $P$ and the $N_{L}$ currents, the electric field gradient reverses, but not the sign of the electric field. The $N_{L}$ current is associated with the electric field gradient alone, since the conductance is almost constant in this region. As shown by relation (6), the electric field gradient is proportional to the parallel current, under these circumstances. It is fairly large in the $0.5^{\circ}$ latitudinal range where the parallel current is large $\left(1.5 \mu \mathrm{A} \mathrm{m}^{-2}\right)$. In conclusion, the arc has a large longitudinal extent and presents a central positive current with negative parallel currents on both sides. Electric field gradients drive the negative currents, while, on the opposite, the conductance gradient on the equatorward side of the main precipitation drives the $P$ current.

The similarity between modelled and experimental profiles of the east-west convection velocity along the FAST orbit also supports the latitudinal one-dimensional hypothesis. The FAST trajectory is rather in the latitudinal part of the convection pattern, at the east of the vortex. These results are confirmed by the fact that the direction of the three current sheets detected by FAST are almost parallel to the magnetic east-west direction (Table 1). The 1-D model is very efficient in the case of a purely latitudinal convection pattern, as shown by the results obtained along the FAST orbit. Figure 11 shows the instantaneous radial velocity maps of the two CUTLASS radars, at the time of the FAST pass. These maps will be used for comparing the experimental and modelled east-west convection profiles. Although the Pikkvibær map contains large data gaps, several patches of eastward velocities above $69^{\circ}$ MLAT (red and orange velocities) and several patches of westward velocities (blue) below $69^{\circ}$ MLAT are observed. This convection reversal agrees well with the convection shear observed around $68.5^{\circ}$ MLAT by FAST. The Hankasalmi map is more detailed, but the velocity component measured with this radar is predominantly in the north-south direction. As the convection velocities are mainly east-west, the Hankasalmi map does not provide the most significant component of the convection. However, several features of this map confirm the results of the modelling. Exactly on the FAST trajectory, an enhancement of the eastward convection is observed (a small east-west band of orange and yellow velocities), exactly at the latitude of the strong peak of eastward velocities measured by FAST, at $73^{\circ}$ MLAT. Assuming a purely longitudinal flow, this lineof-sight velocity turns into a flow velocity of $\sim 2000 \mathrm{~m} \mathrm{~s}^{-1}$, a value which agrees quantitatively with the modelled result of $1700 \mathrm{~m} \mathrm{~s}^{-1}$ (Fig. 8). Further south, FAST crosses a region of predominantly eastward but smaller velocities and is tangent to a complex structure of the radial velocities corresponding to a strong decrease in the velocity and associated with the gradient reversal measured by FAST, at $71^{\circ}$ MLAT. Then, at about $69^{\circ}$ MLAT, a real east-west convection shear is observed associated with the convection reversal also observed on the Pikkvibær map and on the FAST east-west convection profiles. These similarities between FAST and SuperDARN confirm the validity of the modelled east-west convection profile obtained with the 1-D model.

\section{2 Ørsted results}

Along the Ørsted orbit, the 1-D hypothesis is less justified than in the FAST case. First, the SuperDARN convection map (Fig. 4a) shows that the Ørsted trajectory is located in the vortical part of the convection structure contrary to the FAST trajectory. Then, the 3 sheets of parallel current detected by Ørsted have very different orientations, which do not follow the magnetic isolatitude direction as expected with the 1-D latitudinal hypothesis. A rotation of the sheet directions is observed, supporting the vortical structure of the convection (Table 1). Moreover, magnetometer data from the IMAGE chain show that the directions of the equivalent Hall currents in the vortical part of the convection structure are also very different from the magnetic isolatitude direction. From north to south, a rotation of their direction opposite to the convection vortex is observed, as expected. All these considerations allow one to conclude that the 1-D model is not adapted to the Ørsted conjunction and can explain why the modelled conductance becomes negative in the $N_{L}$ cur- 


\section{SUPERDARN PARAMETER MAP}
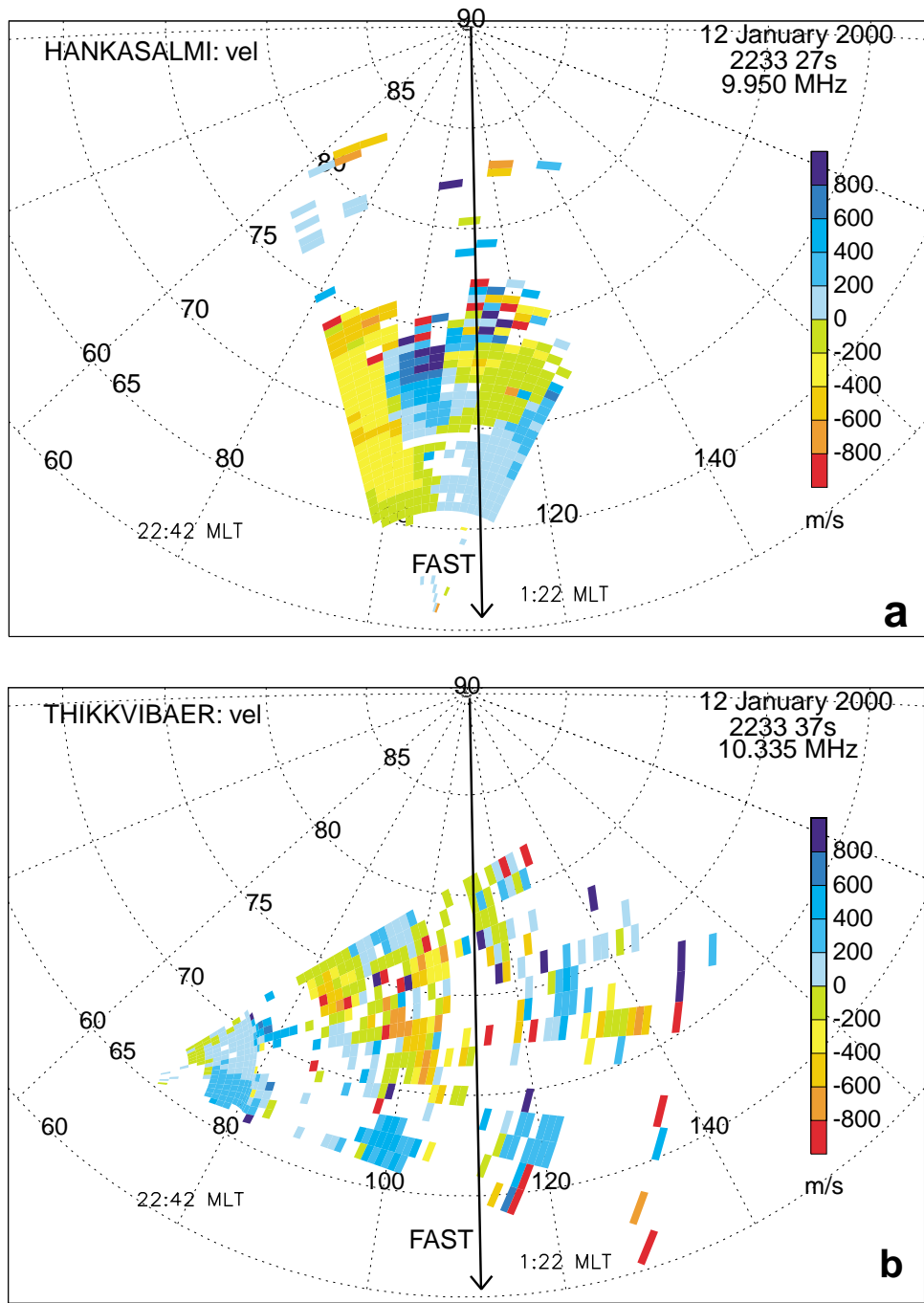

Fig. 11. Line-of-sight velocity maps of the Pikkvibær (top panel) and Hankasalmi (bottom panel) radars in AACGM coordinates, at the time of the FAST pass, with the FAST orbit superimposed. rent of the vortex. This is why we have used a 2-D model to obtain more realistic results of the conductance profile along the Ørsted orbit.

However, the FAC input to the 2-D model is known only along the Ørsted orbit and has to be extrapolated elsewhere. In particular, the density of the central part of the $P$ current is chosen to be constant, which is probably not the case. This incorrect description of the centre of the $P$ current can explain the unexpected small values of conductance located in the vicinity of the vortex centre. For these reasons, the conductance profile deduced from this 2-D model is expected to be more accurate close to the Ørsted trajectory than far from it, because the FAC evaluation is also less acurate with increasing distance from the Ørsted orbit. Thus, the 2-D modelling also presents insufficiencies but allows for a correct description of the conductance along the Ørsted orbit.

If we compare the results of the conductance profile obtained with the 1-D and the 2-D models along the Ørsted orbit, we note several differences. The highest conductance ob- tained with the 2-D model $(8 \mathrm{~S})$ is slightly smaller than with the 1-D model $(10.5 \mathrm{~S})$, which can be explained by the spatial smoothing involved. Moreover, with the 2-D model, the spatial extent of the maximum of conductance is large and coincides with the $P$ current, contrary to the 1-D model, for which the maximum of conductance is narrow and located at the boundary between the $P$ and $N_{H}$ currents, with the $P$ current being associated with a conductance gradient. This last result seems to better support the 2-D model, because it is more likely that in an arc maintained by FACs, the downward electron precipitation associated with the conductance increase is coupled with the centre of the upward current and that the conductance gradients are located at the boundaries of the upward current (Burch et al., 1976; Opgenoorth et al., 1990). In spite of the above differences, both models give essentially the same global elongated shape of the precipitation pattern.

The two models present limitations inherent to the assumed hypothesis: the latitudinal structure in the 1-D case 


\section{2:15 UT}

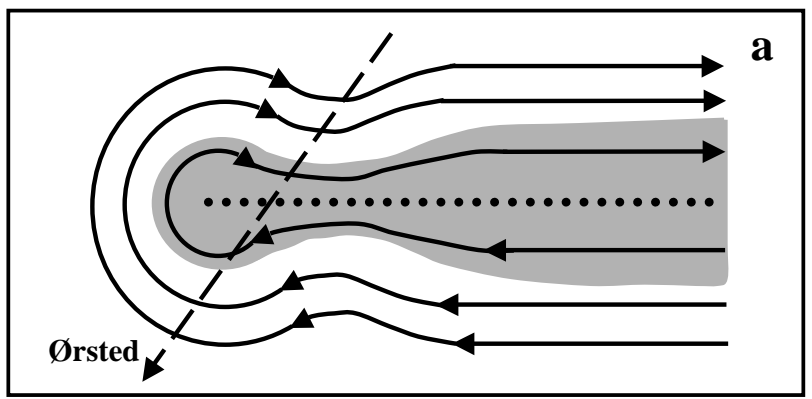

\section{2:35 UT}

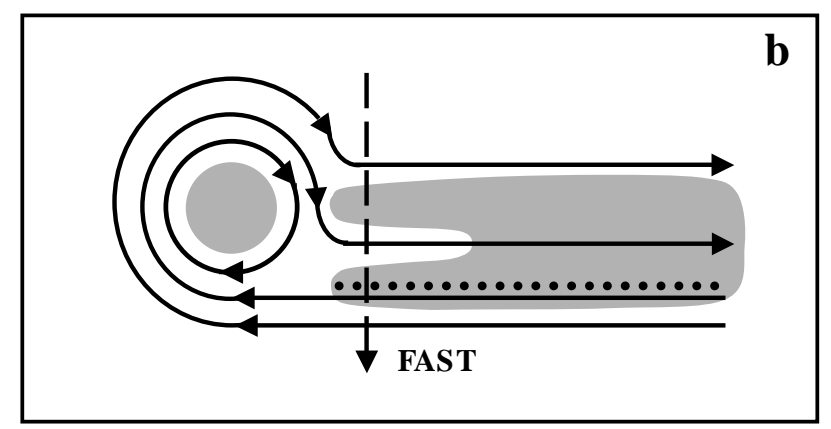

Fig. 12. Schematic representations of the ionospheric precipitation (grey surface) and convection pattern (solid lines) with the convection velocity shear superimposed (dotted line). (a) At the time of the Ørsted pass with the Ørsted trajectory superimposed (dashed line). (b) At the time of the FAST pass, with the FAST trajectory superimposed (dashed line).

and the need to model the 2-D parallel current pattern from 1 -D satellite measurements in the 2-D case. Although the validity of these hypotheses cannot be checked from the experimental data set, they are probably not fully justified, as shown by the insufficiencies of the results. However, these two models are the best which can be constructed from this data set. The results of both models show similarities confirming the general structure of the precipitation pattern during the Ørsted pass (described precisely in the next subsection).

\subsection{Time evolution}

This double conjunction can be used to understand the time evolution of the current and precipitation patterns between the Ørsted and FAST passes. First, a global northward motion of the structure is observed between the two satellites passes. Thus, the triple current-sheets system has been shifted northward by about $1^{\circ}$ MLAT during the 20 min-time interval. The precipitation region associated with the $P$ current is also shifted northward. Moreover, strong modifications of the convection-precipitation pattern are also observed between the two satellites passes. In both cases, the Polar images show a continuous band of precipitation in the early morning sector, extending eastward to the limit of the field of view at about 03:00 MLT. Both satellites' passes occur on the western edge of this precipitation where the pattern modifications occur. Figure $12 \mathrm{a}$ and $\mathrm{b}$ show schematic representations of the convection-precipitation pattern at the times of the Ørsted and FAST passes, deduced from the SuperDARN convection maps (Fig. 4a, b) and from the Polar-UVI images (Fig. 3b, c). During the Ørsted pass (Fig. 12a), the main region of precipitation (grey surface) has a quasi-continuous east-west extent limited westward of around 23:30 MLT and is associated with the semi-elliptical clockwise vortex (solid lines) also having mainly a large eastwest extent and closed on the western side. However, a clockwise rotation of the vortex is observed with respect to the main region of precipitation located at the east and also a slight narrowing of the precipitation region just eastward of the Ørsted pass (dashed line). Thus, Ørsted crosses the vortical part of the precipitation-convection pattern which is probably more two-dimensional than the region situated at later MLTs. This justifies the conclusion made in the previous section, that the 1-D model is not adapted to the Ørsted case. At the time of the FAST pass (Fig. 12b), the western part of the precipitation (grey surface) has separated from the main precipitation to form a single isolated spot associated with the circular convection vortex at the west (solid lines). An intensification of southward velocities is then observed to close the eastern side of the vortex and keep it isolated, supporting the idea, that this part of the convection pattern is now fully two-dimensional compared to the eastward part of the precipitation which keeps the purely latitudinal structure. Thus, this eastward precipitation forms a double purely latitudinal structure with a gap at the centre where the $N_{L}$ current is observed, supported by the purely east-west convection lines in this region. FAST crosses this second part of the precipitation, which explains why the one-dimensional model is valid along its trajectory.

Finally, we can also note a strong $3^{\circ}$ MLAT southward shift of the convection velocity shear between the two satellites passes (dotted line of Fig. 12a and b). Thus, at the time of the FAST pass, the arc is no longer associated with the east-west convection shear as observed along the Ørsted orbit and generally in previous studies (Gurnett and Frank, 1973; Burch et al., 1976; Taguchi, 1992; Taguchi et al., 1994), but simply with a reversal of the gradient in the east-west convection velocity. These observations imply that the system of 3 field-aligned current sheets is not necessarily associated with a convection shear, in order to maintain the divergence of the Pedersen currents.

All these observations show that the nightside ionosphere is dynamic, in spite of the quiet magnetic conditions characterised by a positive IMF $B_{z}$. And consequently, the triple current-sheets system of Taguchi (1992), associated with positive $B_{z}$ and negative $B_{y}$ of the IMF, is not static. Thus, the currents' systems observed by FAST and Ørsted are very different because of the time evolution and because the two satellites detect the currents in different parts of the precipitation. This is why the success of the simulations are very 
different between Ørsted and FAST.

Is the current system balanced? We have checked the balance of the 3 current-sheets system. Based on the 1-D latitudinal profile of the current structure along the FAST orbit, we have integrated the total positive and negative latitudinal currents. The total positive current is $0.294 \mathrm{~A} \mathrm{~m}^{-1}$ and the sum of the two negative currents is $-0.309 \mathrm{~A} \mathrm{~m}^{-1}$, showing that positive and negative currents are almost equal. Thus we can conclude that the 3 current-sheets system is balanced and isolated from the rest of the auroral oval. Along the Ørsted orbit, the 3 current-sheets system is not one-dimensional and consequently, the balance between negative and positive currents cannot be verified.

\subsection{Magnetospheric coupling}

The simultaneous appearance of high energy population (up to $1 \mathrm{keV}$ ) in the ion flux and of the inverted-V structures in the downgoing electrons' diagram associated with the $P$ current of the 3 current sheets suggests that this structure is localised on the boundary between opened and closed field lines, but is, however, essentially on closed field lines. This structure has its magnetospheric origin in the magnetotail, probably in the Plasma Sheet Boundary Layer (PSBL), since the ions' and electrons' high mean energies and the inverted$\mathrm{V}$ structures of the electron precipitation are typical of this magnetospheric region (Eastman et al., 1984; Huang et al., 1984).

Another feature related to the magnetospheric origin of the particles reveals the dynamical situation of the nightside ionosphere, already noted in the General Context section. Between about 22:00 and 00:00 UT, intensifications of the electron precipitation are observed every $6 \mathrm{~min}$ in the whole nightside ionosphere. Ørsted and FAST cross the auroral oval just before a maximum and during a minimum of the precipitation, respectively. This probably explains the larger values of the parallel currents during the Ørsted pass than during the FAST pass. The periodic intensifications of the precipitation pattern are probably due to regular injections of particles associated with reconnection in the magnetotail.

\section{Summary}

Two successive conjunctions between the CUTLASS SuperDARN radars and the Ørsted and FAST satellites occur in the nightside ionosphere on 12 January 2000, between 22:10 and 22:40 UT, and around 00:00 MLT. The Polar-UVI satellite and the IMAGE magnetometer data are also available in this region, at the same period. The large data set obtained during these conjunctions allows one to understand the structure and the time evolution of a nightside arc associated with a convection shear, during relatively quiet interplanetary conditions (stable IMF $B_{z}>0$ and $B_{y}<0$ and stable solar wind pressure). Three field-aligned currents, one upward and two downward on each side, in order to close the global current system, are observed by the two satellites.
Modelling of the arc along the two satellites passes, based on the current continuity equation, allows one to understand the association between the electric field and conductance gradients which are the source of parallel currents. Moreover, the 20-min time lag between the two satellites' passes and the time series of SuperDARN maps and Polar images, allow one to describe the evolution of the nightside arc. Periodic enhancements of the convection and electron precipitation associated with the arc are observed, probably associated with regular, periodic injections of particles due to reconnection in the magnetotail. A general northward motion of the electron precipitation and field-aligned currents is observed between the two satellites' passes. In addition, a reorganization of the precipitation and of the convection patterns in the vicinity of the arc occur, characterised by a dissociation of the electron precipitation into a single spot at the western edge of the arc, whereas the convection shear at the eastern side is shifted by $3^{\circ}$ MLAT southward implying that the arc and the associated system of 3 field-aligned current sheets are no longer associated with the convection shear, in order to maintain the divergence of the Pedersen currents.

Acknowledgements. The authors acknowledge M. Berthomier for helpful discussions concerning the FAST data interpretation. The CUTLASS HF radars are deployed and operated by the University of Leicester, and are jointly funded by the UK Particle Physics and Astronomy Research Council (grant number PPA/R/R/1997/00256), the Finnish Meteorological Institute, and the Swedish Institute of Space Physics. The Ørsted satellite project is funded by the Danish Ministry of Research and Information Technology, the Ministry of Trade and Industry, and the Danish Research Councils. The Ørsted satellite is operated by TERMA Electronics and the Danish Meteorological Institute (DMI). FAST data analysis at the University of California in Berkeley was supported through NASA grant NAG5-3596. The authors acknowledge the principal investigator N. Ness and the ACE Science Center for providing the ACE magnetic field data and the Finnish Meteorological Institute for providing the IMAGE data.

The Editor in Chief thanks A. D. M. Walker and A. Aikio for their help in evaluating this paper.

\section{References}

Aikio, A. T., Lakkala, T., Kozlovsky, A., and Williams, P. J. S.: Electric fields and currents of stable drifting auroral arcs in the evening sectors, J. Geophys. Res., 107, 1424, doi:10.1029/2001JA009172, 2002.

Aikio, A. T., Marklund, G. T., Woch, J., and Potemra, T. A.: Smallscale structures in the high-latitude auroral electric field, Ann. Geophysicae, 13, 84-94, 1995.

Aikio, A. T., Opgenoorth, H. J., Persson, M. A. L., and Kaila, K. U.: Ground-based measurements of an arc-associated electric field, J. Atmos. Terr. Phys., 55, 797-808, 1993.

Amm, O.: Direct determination of the local ionospheric Hall conductance distribution from two-dimensional electric and magnetic field data: Application of the method using models of typical ionospheric electrodynamic situations, Geophys. Res. Lett., 100, 21 473-21 488, 1995. 
Amm, O.: Ionospheric elementary current systems in spherical coordinates and their application, J. Geomag. Geoelectr., 49, 947, 1997.

Amm, O.: Method of characteristics for calculating ionospheric electrodynamics from multisatellite and ground-based radar data, J. Geophys. Res., 107, 1270, 2002.

Amm, O.: Method of characteristics in spherical geometry applied to a Harang Discontinuity situation, Ann. Geophysicae, 16, 413424, 1998

Baker, K. B. and Wing, S.: A new magnetic coordinate system for conjugate studies at high latitudes, J. Geophys. Res., 94, 91399143, 1989.

Burch, J. L., Lennartsson, W., Hanson, W. B., Heelis, R. A., Hoffman, J. H., and Hoffman, R. A.: Properties of spikelike shear flow reversal observed in the auroral plasma by Atmosphere Explorer C, J. Geophys. Res., 81, 3886-3896, 1976.

Carlson, C. W., Pfaff, R. F., and Watzin, J. G.: The Fast Auroral SnapshoT (FAST) mission, Geophys. Res. Lett., 25, 2013-2016, 1998.

De la Beaujardière, O., Lyons, R., Ruohoniemi, J. M., FriisChristensen, E., Danielsen, C., Rich, F. J., and Newell, P. T.: Quiet-time intensifications along the poleward auroral boundary near midnight, J. Geophys. Res., 99, 287-298, 1994.

De la Beaujardière, O., Vondrak, R., and Baron, M.: Radar observations of electric fields and currents associated with auroral arcs, J. Geophys. Res., 82, 5051-5062, 1977.

Eastman, T. E., Franck, L. A., Peterson, W. K., and Lennartsson, W.: The Plasma Sheet Boundary Layer, J. Geophys. Res., 89, 1553-1572, 1984.

Freeman, M. P, Farrugia, C. J., Burlaga, L. F., Hairston, M. R., Greenspan, M. E., Ruohoniemi, J. M., and Lepping, R. P.: The interaction of a magnetic cloud with the Earth: Ionospheric convection in the northern and southern hemispheres for a wide range of quasi-steady interplanetary magnetic field convection, J. Geophys. Res., 98, 7633-7655, 1993.

Greenwald, R. A., Baker, K. B., Dudeney, J. R., Pinnock, M., Jones, T. B., Thomas, E. C., Villain, J.-P., Cerisier, J.-C., Senior, C., Hanuise, C., Hunsucker, R. D., Sofko, G., Koehler, J., Nielsen, E., Pellinen, R., Walker, A. D. M., Sato, N., and Yamagishi, H.: DARN/SuperDARN: A global view of high-latitude convection, Space Sci. Rev., 71, 761-796, 1995.

Gurnett, D. A. and Franck, L. A.: Observed Relationships between electric fields and auroral particle precipitation, J. Geophys. Res., 78, 145-170, 1973.

Hardy, D. A., Gussenhoven, M. S., Raistrick, R., and McNeil, W. J.: Statistical and Functional Representations of the Pattern of auroral energy flux, number flux, and conductivity, J. Geophys. Res., 92, 12 275-12 294, 1987.

Huang, C. Y., Frank, L. A., and Eastman, T. E.: High-altitude observations of an intense inverted V event, J. Geophys. Res., 89, 7423-7430, 1984.
Iijima, T. and Potemra, T. A.: Field-aligned currents in the dayside cusp observed by Triad, J. Geophys. Res., 81, 5971-5979, 1976.

Janhunen, P., Olsson, A., Amm, O., and Kauristie, K.: Characteristics of a stable arc based on FAST and MIRACLE observations, Ann. Geophysicae, 18, 152-160, 2000.

Johnson, M. L., Murphree, J. S., Marklund, G. T., and Karlsson, T. Progress on relating forms and electric field patterns, J. Geophys. Res., 103, 4271-4284, 1998.

Kosch, M. J., Amm, O., and Scourfield, M. W. J.: A plasma vortex revisited: The importance of including ionospheric conductivity measurements, J. Geophys. Res., 105, 24 889-24 898, 2000.

Marklund, G.: Auroral Arc classification scheme based on the observed arc-associated electric field pattern, Planet. Space Sci., 32, 193-211, 1984.

Marklund, G., Sandahl, I., and Opgenoorth, H. J.: A study of the dynamics of a discrete auroral arc, Planet. Space Sci., 30, 179197, 1982.

Marklund, G., Baumjohann, W., and Sandahl, I.: Rocket and ground-based study of an auroral breakup event, Planet. Space Sci., 31, 207-220, 1983.

Opgenoorth, H. J., Haggstrom, I., Williams, P. J. S., and Jones, G. O. L.: Regions of strongly enhanced perpendicular electric fields adjacent to aurorals arcs, J. Atmos. Terr. Phys., 52, 449458, 1990.

Senior, C., Cerisier, J.-C., Rich, F. J., Lester, M., and Parks, G. K.: Strong sunward propagating, flow bursts in the night sector during quiet solar wind conditions: SuperDARN and satellite observations, Ann. Geophysicae, 20, 771-779, 2002.

Senior, C.: Solar and particle contributions to auroral heightintegrated conductivities from EISCAT data: A statistical study, Ann. Geophysicae, 9, 449-460, 1991.

Stauning, P., Primdahl, F., Watermann, J., and Rasmussen, O.: IMF $B_{y}$-related Cusp currents observed from the Ørsted satellite and from ground, Geophys. Res. Lett., 28, 99-102, 2001.

Taguchi, S. and Hoffman, R. A.: Ionospheric plasma convection in the midnight sector for northward interplanetary magnetic field, J. Geomag. Geoelectr., 48, 925-933, 1996.

Taguchi, S.: $B_{y}$-controlled field-aligned currents near midnight auroral oval during northward Interplanetary magnetic field, J. Geophys. Res., 97, 12 231-12 243, 1992.

Taguchi, S., Sugiura, M., Iyemori, T., Winningham, J. D., and Slavin, J. A.: $B_{y}$-controlled convection and field-aligned currents near midnight auroral oval for northward interplanetary magnetic field, J. Geophys. Res., 99, 6027-6044, 1994.

Untiedt, J. and Baumjohann, W.: Studies of polar current systems, Space Sci. Rev., 63, 245-390, 1993.

Wahlund, J.-E. and Opgenoorth, H. J.: EISCAT observations of strong ion outflows from the F-region ionosphere during auroral activity: preliminary results, Geophys. Res. Lett., 16, 727-730, 1989. 